\title{
Motion of Star-Branched Chains in a Nanochannel. A Monte Carlo Study
}

\author{
Piotr Romiszowski*, Andrzej Sikorski \\ Department of Chemistry, University of Warsaw \\ ul. Pasteura 1, 02-093 Warsaw, Poland \\ e-mail:prom@chem.uw.edu.pl \\ (Received: 17 February 2012; revised: 09 June 2012; accepted: 11 June 2012; published online: 26 June 2012)
}

\begin{abstract}
In order to determine the structure and dynamic properties of polymers systems in a random environment we developed and studied an idealized model. Properties of the model of confined linear and branched polymer chains were studied by means of the Monte Carlo method. Model chains were built of statistical segments and embedded to a simple cubic lattice. Then, the polymers were put into a tube formed by four impenetrable surfaces. A Metropolis-like sampling Monte Carlo algorithm was used to determine the static and dynamic properties of these model macromolecules. The influence of the size of the confinement (the tube diameter) and the chain length on polymer properties was studied. The universal behavior of confined polymer linear chains under consideration was found and discussed. The long-time (diffusion) dynamic properties of the system were also studied. The differences in the mobility of chains depending on the number of branches was shown and discussed - stars with an even number of arms exhibited the ballistic motion at certain conditions. The possible mechanism of the chain's motion was discussed.
\end{abstract}

Key words: branched polymers, lattice models, Monte Carlo method, porous media

\section{INTRODUCTION}

The polymer systems in confined space are the subject of many studies because of their practical importance in lubrication, adhesion, separation methods based on the capillary electrophoresis, corrosion protecting coating or colloidal stabilization [1]. These systems are difficult to be studied experimentally although recently an enormous progress has been made [2]. The influence of the confinement on the properties of polymer or protein chains is especially interesting from the theoretical point of view, as the presence of an impenetrable or attractive wall can change the properties of such systems dramatically. The understanding and describing of such changes is an interesting and challenging problem [3].

Recently, a lot of computer simulation studies were published on confined polymer systems [4-8]. It was shown that the dynamic properties of confined polymer systems are significantly different from those of bulk polymers. The static and dynamic properties, their scalings, distribution of segments, the internal structure of chains and instantaneous shape were intensively studied and discussed. The influence of the internal macromolecular architecture (linear chains, star-branched chains, cyclic chains) on the properties of confined polymers was also studied employing idealized lattice models and computer simulation [9-11]. The short- and long-time dynamics, orientation and deformation of coils and instantaneous shape of the confined chains were also determined. The confinement theoretical treatment of a polymer chain in a tube was also a subject of interest for computer simulations [12-17] and theoretical considerations [18-20]. The structure of the chain compressed in a narrow tube (narrow means that the diameter of the tube is smaller than the diameter of the polymer chain at good solvent conditions) was determined and compared to that of chains in a bulk solution. It was shown that the size of the linear chain in a tube obeys the following scaling relations:

$$
S_{\|}^{2} \sim N^{2} d^{-4 / 3}
$$

where $S_{\|}^{2}$ is the mean-squared radius of gyration in the direction along the tube axis, $d$ is the diameter of the tube and 
$N$ is the length of chain (the number of statistical segments). The lateral part of the radius of gyration $S_{\perp}^{2}$ reaches the plateau very quickly as the size in this direction is limited by the radius of the tube. It scales as $N^{2 v}$ for short chains only and the scaling exponent $2 v=1.18$ is characteristic for 3-dimensional chains. Therefore, a chain in a tube has the same fractal dimension as stiff elongated objects (rods, needles). The lateral motion of the chain in the tube was suppressed while the self-diffusion coefficient along the tube was found to scale as in dilute 3-dimensional solutions but additional dependence on the diameter of the tube had to be added:

$$
D_{\|} \sim d^{-2 / 3} N^{-1}
$$

The experiments concerning polystyrenes in microporous membranes confirmed this scaling behavior [21]. The scaling exponent of a linear chain in a tube is therefore the same as for diluted polymer solutions.

In this paper we present the results of computer simulations of simplified models of confined branched chain systems. The main goal of this study was to determine the mechanism of motion and the dynamic properties of chain with different internal architectures. For this purpose we designed a coarse grained model of chains embedded to a simple cubic lattice. The polymers were modeled at good solvent conditions and their properties were determined by means of the Monte Carlo simulations with the sampling algorithm of a Verdier-Stockmayer type using local modifications of chain's conformation.

\section{THE MODEL}

\section{AND THE SIMULATION MODEL}

A polymer chain was in our model reduced to a sequence of identical segments (a segment corresponded to some chemical mers). We studied linear and star-branched chains. Each linear chain consisted of the sequence of $N$ beads connected by $N-1$ segments [11]. The star branched chain consisted of $f$ identical linear chain consisting of $n$ beads and emanating from a common origin called the branching point. The total number of beads in the star was $N=f \cdot(n-1)+1$. In order to make the calculation more efficient the chains were embedded to a simple cubic lattice. We studied the properties of a chain as a whole without details on the level of a single bead so this lattice approximation was quite sufficient for this purpose [12]. The excluded volume interactions were introduced into the model by forbidding the chain to cross itself what corresponds to good solvent contitions. No intrapolymer long-range attractive potential was added and chains were fully flexible with no local stiffness introduced. The macromolecules were put into a tube formed by two pairs of impenetrable parallel planar surfaces. The distance between these surfaces $d$ was varied (there were $d$ unoccupied lattice sites between walls in the direction perpendicular to each pair of walls). The confining walls were impenetrable (no attractive interactions with chains) and thus their influence was entropic only.

The properties of model systems were determined by means of the Monte Carlo simulation. We employed a sampling algorithm that consisted of local random modifications of chain's conformation [9]. This algorithm was found efficient for single chains in neutral and attractive slits [10-11]. The following local modifications of chain's conformation were applied: 1-bead motion, 2-bead motion, 2-bead crankshaft motion, 1-bead end of chain reorientations and 2-bead end of chain reorientations [9]. Star-branched chains required an additional move that involve a collective displacement of the vicinity of the branching point [22]. A new conformation was accepted due to geometrical constraints and the excluded volume condition. All types of these elementary moves were employed with the same frequency because such a procedure was efficient and provided the correct time scale in the simulation $[9,22]$. The time unit was defined as one attempt of each type of local motions per polymer bead on average.

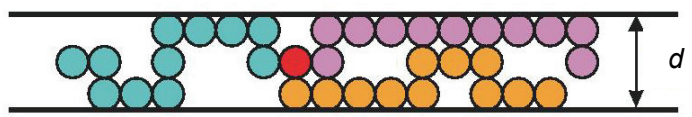

Fig. 1. A typical conformation of a 3-arm star-branched chain in a narrow nanochannel - here is the case for $d=3$. Each chain arm is in different color, branching point is in red

Each simulation run consisted of $10^{9}-10^{10}$ time units and it started from a different initial chain conformation. At the start of each simulation run the equilibration run was performed, which lasted $10^{7}-10^{8}$ time units. The criterion of the equilibration of the system was the stability of some timemean parameters of the system, such as radius of gyration and the single-bead autocorrelation function. For each system under consideration we performed 20-30 independent simulation runs using different initial configurations.

\section{RESULTS}

The simulations were performed for linear, 3-armed and 4-armed branched chains confined in narrow nanochannels with the diameter $d=3,4,5,6$ and 7 lattice units. The 
model star-branched chains with 3 arms were the main object of our study and we determined their properties for a wide range of polymer length with $N=49,100,199,400$, 799 and 1200 beads.

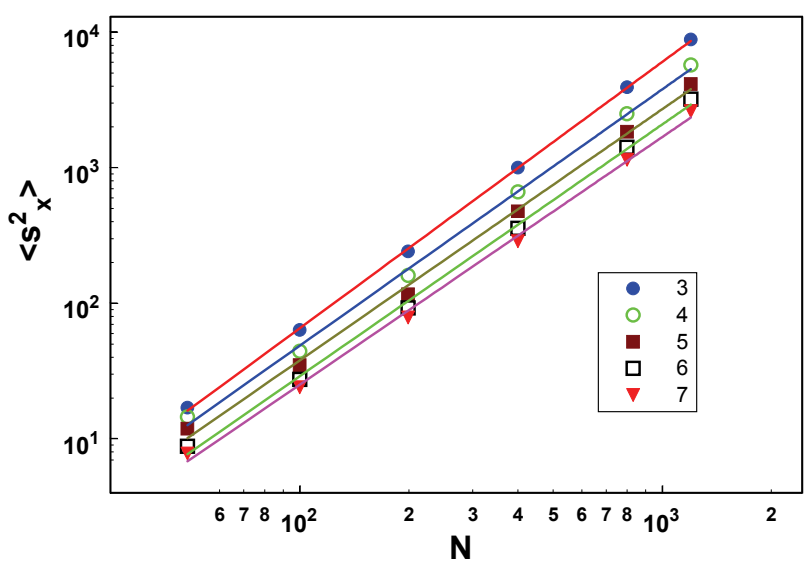

Fig. 2. The mean-squared radius of gyration parallel to the tube's axis $<S^{2}{ }_{x}>$ as a function of the chain length $N$. The diameter of the nanochannel $d$ is given in the inset

In Figure 2 we present the $x$-component (along the tube axis) of the mean-squared radius of gyration $\left\langle S^{2}{ }_{x}>\right.$ that was calculated for different chain length $N$ and for various tune diameters $d$. These results concern star-branched chains with $f=3$ branches. One can observe that the plots for different values of $d$ form straight line, which implies a scaling behavior $S^{2} \sim N^{2 v}$ with the scaling exponent $2 v$ varying from $1.83(d=7)$ to $1.96(d=3)$. These values show that in a narrow tube $\left\langle\mathrm{S}_{\mathrm{x}}^{2}\right\rangle$ scales as a stiff elongated object (rod, needle) where the exponent $2 v=2$ is expected. The same scaling behavior was predicted and found in simulations of linear chains $[12,18]$. For wider

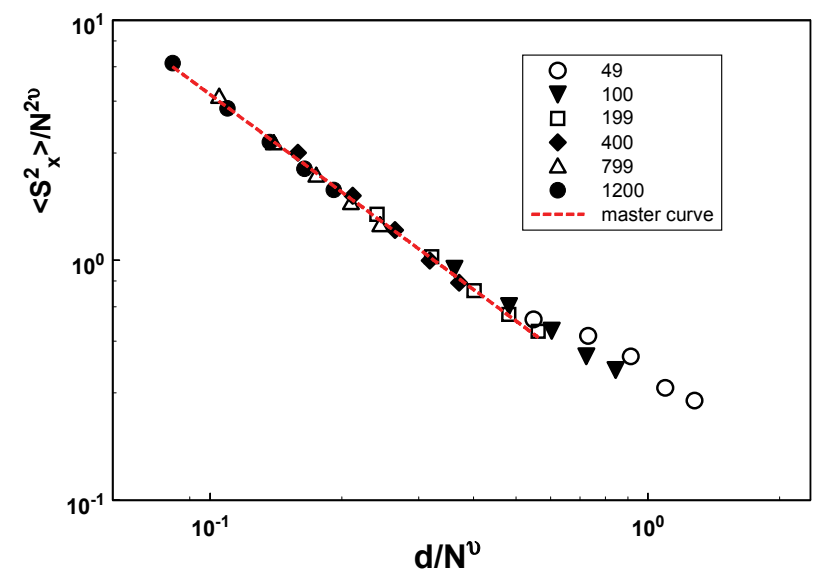

Fig. 3. Plot of $<S^{2}{ }_{\mathrm{x}}>/ N^{2 v}$ with $d / N^{v}$ for 3-branched chains. The points are taken from the simulations performed for different nanochannel diameters $d$ and chain length $N$ (given in the inset) tubes the value of the exponent decreases; for $N=49$ and $d=7$ the diameter of the chain becomes comparable with the tube diameter.

In Figure 3 we present the universal behavior of the $<S^{2}{ }_{x}>$. Here we plot the reduced value of the radius of gyration $<S^{2}{ }_{x}>/ N^{2 v}$ as the function of the reduced tube diameter $d / N^{v}$. The universal scaling behavior results in a master curve regardless the chain length and the tube diameter with the exception the shortest chains $(N=49$ and $100)$, which had the values $\left\langle S^{2}{ }_{x}>\right.$ comparable with $d$. The slope of this master curve was found as -1.36 . This result is in full agreement with that theoretically predicted $-4 / 3$ [12] and -1.22 [18] and with the simulations results [12].

The problem which we tried to focus on this work concerned the dynamic behavior of polymers in the tube. The long-time dynamics of the chain is usually discussed in terms of the autocorrelation function of the center of mass $x$-component, which is defined as

$$
g_{c m}(t)=\left\langle\left(x_{c m}(t)-x_{c m}(0)\right)^{2}\right\rangle
$$

where $x_{c m}$ is the position of the center of mass taken from the simulation trajectory. We have also calculated a single bead autocorrelation function defined as:

$$
g(t)=\frac{1}{N} \sum_{i=1}^{N}\left\langle\left(x_{i}(t)-x_{i}(0)\right)^{2}\right\rangle
$$

where $x_{i}$ is the position of $i$-th bead.

The dynamics of the chains was analyzed from the trajectories in which the positions of all beads during the simulation were recorded. In Figure 4 we present the results concerning both autocorrelation functions obtained for linear chain $N=50$ and the branched chains with $f=3$ and $f=4$ arms $(N=199$ and $N=201$ respectively) for comparison. The plots of the autocorrelation function $g_{\mathrm{cm}}(t)$ definitely have a different slope - the linear and 4-armed chains exhibit the scaling typical for a Fick-type diffusion $g_{c m}(t) \sim t^{l}$ while the 3 -armed chain was moving along the nanochannel in a ballistic motion regime, i.e. with the scaling $g_{c m}(t) \sim t^{2}[23]$. Please note that the simulations for the 4-armed chain were performed in a nanochannel with the diameter $d=4$ because the smaller values of $d$ did not allow any motion of this type of chain. The result of the test was not surprising since in the case of a linear chain any of two possible directions of motion was equally possible. The case of 4-armed chain is slightly different the presence of 2 arms on each side of the molecule makes the problem symmetrical and no ballistic motion is expected. The only possibility leading to such effect would be a 1-3 distribution of the arms, but it is rather unlikely due to the high crowding in a very narrow nanochannel and 
thus no micromodifications of the chain's conformation could be possible. One should also notice that the mobility of chain depends on the tube diameter rather weakly. This behavior was also predicted by the scaling considerations and simulations for linear chains [12]. One can see that at short times the $g_{c m}$ function scales as $t^{\alpha}$, where $\alpha<1$. This effect is probably caused by the fact that one deals with the coupling of the center of mass motion and the internal relaxation of the chain. One should take into consideration that the above-mentioned motions take place in the lattice model and thus the description of short time effects can be misleading [9]. The behavior of the single bead autocorrelation function describes the short-time behavior of the chain. It was found that it scales with exponent equal to 0.5 which is in perfect agreement with that predicted by the Rouse theory and confirmed in simulations of free chains $[9,22]$. At longer times this function scales with the same exponent as $g_{c m}$ does.

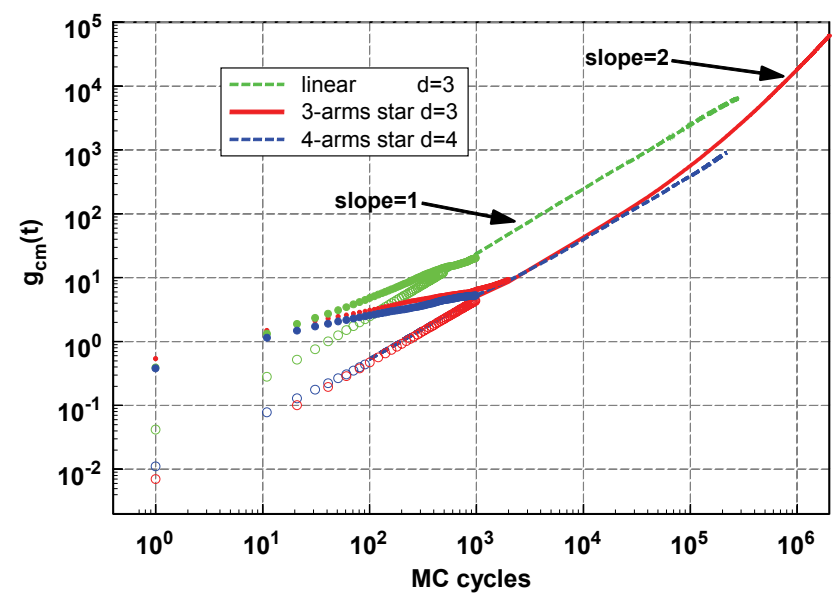

Fig. 4. The center-of-mass autocorrelation functions (open circles for short times and solid lines for longer times) and the single bead autocorrelation functions (solid circles). The macromolecular architectures and diameters of the nanochannel are given in the inset

In further investigation of the dynamics of branched chains in nanochannels we performed the simulations using the 3-armed branched chain consisting of $N=199$ beads confined in nanochannels of different diameters $d=3,4,5$, 6 , and 7 . The results showing the autocorrelation function $g_{c m}(t)$ are presented in Figure 5. One can notice that the autocorrelation function $g_{\mathrm{cm}}(t)$ shows different course depending on value $d$ used in the simulation. In all cases the function shows the scaling with exponent 1 , then at the region $2 \times 10^{5} \mathrm{MC}$ steps the autocorrelation function for the narrowest nanochannel $(d=3)$ changes its slope from 1 to 2, which means that the observed motion of the chain exhibits a characteristic ballistic scaling [23]. That type of motion is not visible for the cases of wider diameter $d$ of the nanochannels.

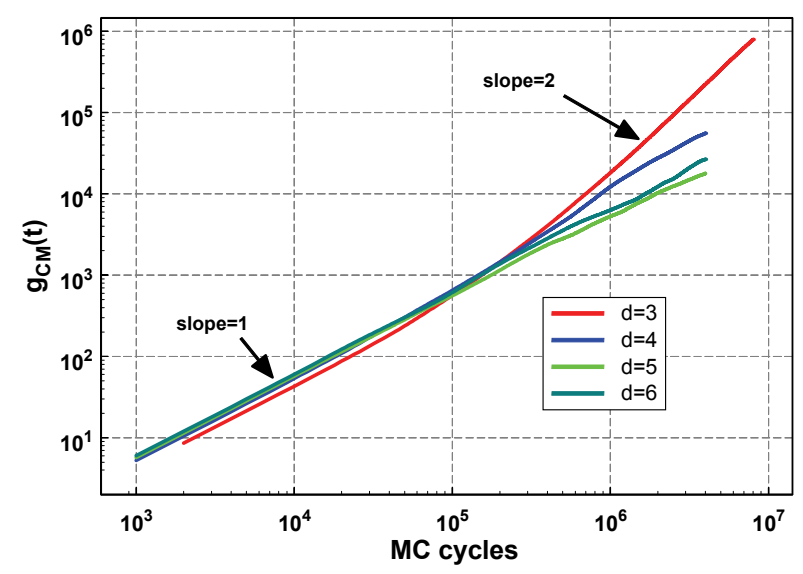

Fig. 5. The center-of-mass autocorrelation function $g_{c m}(\mathrm{t})$ for the star branched chains with $f=3$ arms consisted of $N=199$ beads. The diameter of the nanochannel is given in the inset

In order to take a closer look on the mechanism of motion in narrow nanochannels we have analyzed a typical trajectory recorded during the simulation. The object of the simulation was a 3-armed chain consisting of $N=199$ beads in a nanochannel with diameter $d=3$. The obtained trajectory is plotted in Figure 6. The left $y$-axis shows the position of the branching point along the length of the nanochannel in lattice units. One can see that the chain migrates along the nanochannel towards the negative values of $x$ coordinates; however, from time to time it changes the direction of motion and goes in the opposite direction. Thus, a question arises whether: the mechanism of that motion can we determined. In order to answer this question we made another plot, which was combined with the previous one. The procedure was as follows: from the trajectory we determined the number of beads located at positions with respect to the $x$ coordinate of the branching point counting as "positive" the beads located at $x$-coordinates greater than the position of the branching point and "negative" in opposite direction. The calculations were performed for each arm of the chain. The algebraic sum of these numbers gave the results which are plotted in the right $y$-axis of the plot in Figure 6. One can notice that the two plots combined in one figure correspond almost perfectly - the point in which the trajectory changes its direction is accompanied by the immediate changes in the second plot (see point at abscissa 20000 , for example). The 


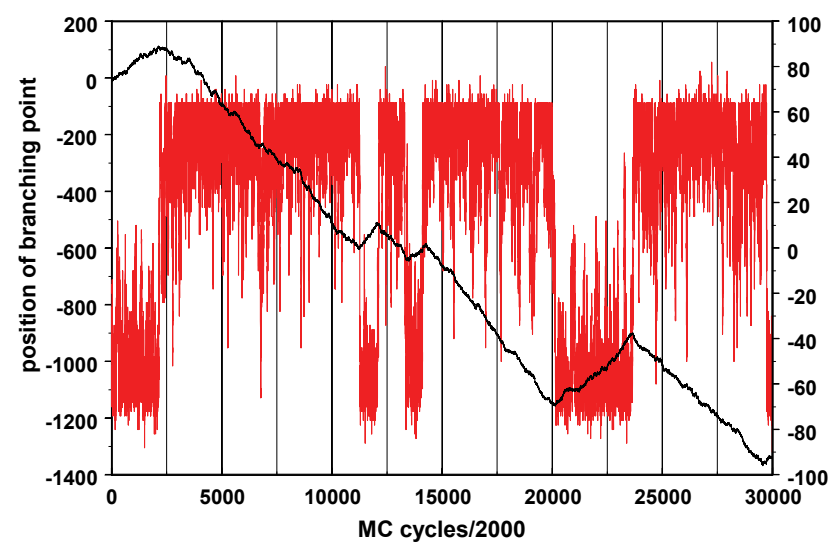

Fig. 6. The trajectory of the branching point of star-branched chains with $f=3$ consisted of $N=199$ beads in a tube $d=3$ (left axis). The number of beads located to the right $(+)$ and to the left $(-)$ with respect to the branching point along the $x$-axis as a function of the time is presented on the right axis - see text for details

described experiment gave us the hint to determine the mechanism of motion of the 3 -armed chain. The direction of motion is governed by the current conformation of the chain - since the nanochannel is very narrow there is not enough room to form loops and entanglements. One could rather expect close to longitudinal stretching of arms. In such assumption one sees that the motion in a positive $x$ direction is associated with one arm pointed towards the direction of motion while the two remaining arms are left behind the branching point and the 'push' the whole structure towards the positive direction. The nanochannel is still large enough to enable the arm's ends change their position passing from one side of the molecule to the other, changing the direction of the longitudinal motion of the chain. If this mechanism is correct one can say that the force, which makes the ballistic motion originates from the entropy of the two arms, located opposite to the direction of the motion of the chain.

\section{CONCLUSIONS}

The aim of this work was the analysis of the long-time dynamics of star-branched chains in a narrow straight nanochannels. The diameter of the nanochannel was smaller than the diameter of the chain in a bulk and thus the later one was compressed. We use a coarse-grained model of macromolecules which consisted of identical segments and were constructed on a cubic lattice. The properties of the system under consideration were determined by means of the dynamic Monte Carlo method employing an algorithm with local changes of chain's conformation.
The results show that star-branched chains confined in a narrow tube scales exhibit a common behavior. Their size scales with the tube diameter and chain length as it was predicted for linear polymers. The analysis of the dynamics shows that the asymmetric 3-arm branched chains exhibit the ballistic motion of the chain in a very narrow nanochannel whilst in wider channels one deals with the Fick's type diffusion. The experiments with the linear as well as the symmetric 4-branched chain show that in these cases no ballistic motion is present. Thus, we can claim that the ballistic motion takes place in the case of 3-branched chain as the asymmetry of the object (one arm located on one side of the branching point and two arms on the other side) enable the 'one direction' motion only during which the unbalanced distribution of polymer beads generates an entropic force.

\section{Acknowledgment}

The computational part of this work was done using the computer cluster at the Computing Center of the Department of Chemistry, University of Warsaw. This work was supported by the Polish Ministry of Science and Higher Education grant N N507 326536.

\section{References}

[1] E. Eisenriegler, Polymers Near Surfaces (World Scientific, Singapore 1993).

[2] M. Ediger, Annu. Rev. Phys. Chem. 51, 99 (2000).

[3] H.-W. Sun, S. Granick, Science 258, 1339 (1992).

[4] G. ten Brinke, D. Ausserre, G. Hadziioannou, J. Chem. Phys. 89, 4374 (1988).

[5] A. Milchev, K. Binder, Eur. Phys. J. B 3, 477 (1998).

[6] Y. Yoshida, Y. Hiwatari, Molec. Simul. 22, 91 (1999).

[7] D.V. Kuznetsov, A.C. Balazs, J. Chem. Phys. 113, 2479 (2000).

[8] N. Fatkulin, R. Kimmich, E. Fischer, C. Mattea, U. Beginn, M. Kroutieva, New J. Phys. 6, 46 (2004).

[9] J. Skolnick, A. Kolinski, Adv. Chem. Phys. 78, 223 (1990).

[10] A. Sikorski, P. Romiszowski, J. Chem. Phys. 116, 1731 (2002).

[11] A. Sikorski, P. Romiszowski, J. Chem. Phys. 123, 104905 (2005).

[12] K. Kremer, K. Binder, J. Chem. Phys. 81, 6381 (1984).

[13] Y.J. Sheng, M.C. Wang, J. Chem. Phys. 114, 4724 (2001).

[14] K. Avramova, A. Milchev, J. Chem. Phys. 124, 024909 (2006).

[15] J. Kalb, B. Chakraborty, J. Chem. Phys. 130, 025103 (2009).

[16] Z. Li, Y. Li, Y. Wang, Z. Sun, L. An, Macromolecules 43, 5896 (2010).

[17] R.M. Jendrejack, D.C. Schwartz, M.D. Graham, J.J. de Pablo, J. Chem. Phys. 119, 1165 (2003). 
[18] J.L. Harden, M. Doi, J. Phys. Chem. 96, 4046 (1992).

[19] P.G. de Gennes, Scaling Concepts in Polymer Physics (Cornell University Press, Ithaca 1979).

[20] G. Morrison, D. Thirumalai, J. Chem. Phys. 122, 194907 (2005).
[21] D.S. Cannell, F. Rondelez, Macromolecules 13, 1599 (1980).

[22] A. Sikorski, Macromol. Theory Simul. 2, 309 (1993).

[23] M. Schmiedeberg, V. Yu. Zaburdaev, H. Stark, J. Stat. Mech. P12020 (2009).

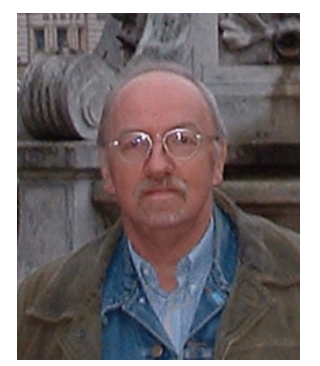

Piotr Romiszowski graduated from the Department of Chemistry, University of Warsaw in 1970. He completed his PhD in the same Department in 1979. He was a post doc in Darthmouth College (1982/83) and in Washington University in St. Louis (1989/91).

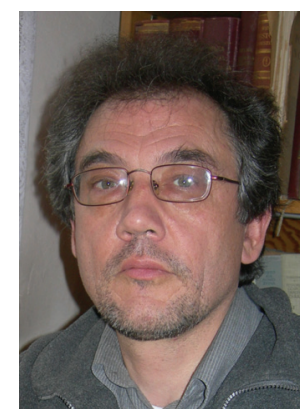

ANDRZEJ SIKORSKI graduated from the Department of Chemistry, University of Warsaw in 1980. He completed his PhD Thesis in Chemistry in 1986 in the same Department. In 1987/89 and 1990/91 he worked as a postdoc and visiting professor in Washington University in St. Louis and in The Scripps Research Institute in La Jolla. In 1995 he obtained DSc degree in Chemistry. His main scientific interest is the theory and simulation of polymers at interfaces and in confined geometries. 